\title{
Reemplazo percutáneo de válvula aórtica en pacientes con estenosis aórtica severa sintomática de riesgo quirúrgico intermedio. Revisión sistemática de estudios clínicos aleatorizados
}

\author{
Percutaneous aortic valve replacement in patients with severe \\ symptomatic aortic stenosis and intermediate risk. Systematic Review \\ from randomized clinical trials
}

A. Matías Rodríguez-Granillo', Carlos Fernández Pereira², Eduardo Gabe ${ }^{1}$

\begin{abstract}
RESUMEN
La estenosis aórtica severa sintomática es la tercera causa más común de enfermedad cardiovascular y se estima que su prevalencia va a ir en aumento. El reemplazo valvular aórtico quirúrgico (RVAO) es el procedimiento de elección indicado para mejorar los síntomas y la sobrevida. El reemplazo valvular aórtico percutáneo (TAVR) es una alternativa menos invasiva en candidatos no quirúrgicos, riesgo quirúrgico alto y comorbilidades. Las nuevas prótesis y la mayor experiencia extendieron su indicación a pacientes con menor riesgo, y la Sociedad Europea de Cardiología y la American Heart Association/American College of Cardiology publicaron recomendaciones sobre la no inferioridad del TAVR vs. la cirugía convencional en pacientes con estenosis aórtica severa sintomática de riesgo intermedio. Solo existe una revisión del tema en nuestro país, del 2014, en donde no se valoran las nuevas evidencias. El objetivo de esta revisión bibliográfica es evaluar los resultados de los nuevos estudios clínicos randomizados y su eventual influencia en nuevas recomendaciones.
\end{abstract}

Palabras claves: estenosis aórtica severa, RVAO, TAVR, riesgo intermedio.

\begin{abstract}
Symptomatic severe aortic stenosis is the third most common cause of cardiovascular disease and it is estimated that the prevalence of the disease will increase. Surgical aortic valve replacement (SAVR) is the procedure of choice indicated to improve symptoms and survival. Percutaneous aortic valve replacement (TAVR) is a less invasive alternative in non-surgical candidates, high surgical risk and comorbidities. The new prostheses and the greater experience, extended their indication to patients with lower surgical risk, and the European Society of Cardiology and the American Heart Association / American College of Cardiology published recommendations of non-inferiority of TAVR vs conventional surgery in aortic stenosis severe symptomatic and intermediate risk. There is only one review of the issue in Argentina in 2014, where the new evidence is not assessed. The objective of this review is to evaluate the results of the new randomized clinical trials and eventually future changes in recomendations.
\end{abstract}

Keywords: severe aortic stenosis, SAVR, TAVR, intermediate risk.

Revista Argentina de Cardioangiología Intervencionista 2018;9(4):204-213. DOl: 10.30567/RACI/201804/0204-0213

\section{INTRODUCCIÓN}

La estenosis aórtica severa sintomática es la tercera causa más común de enfermedad cardiovascular en Europa y Estados Unidos; siendo reconocido el continuo envejecimiento de la población y las comorbilidades de este grupo en crecimiento, se estima que la prevalencia de la enfermedad va a ir en aumento ${ }^{1}$.

El reemplazo valvular aórtico quirúrgico (RVAO) es el procedimiento de elección para mejorar los síntomas y la sobrevida de los pacientes con esta patología ${ }^{1-3}$. En los últimos 15 años, el reemplazo valvular aórtico percutáneo (TAVR, sigla del inglés de transcatheter aortic valve replacement) emergió como una alternativa menos invasiva en pacientes que no eran candidatos quirúrgicos o en aquellos con riesgo quirúrgico alto y comorbilidades $^{4-9}$. La aceptación del uso de la TAVR como alternativa al RVAO se basó en la evidencia a partir de estudios clínicos randomizados que utilizaron dispositivos de generaciones anteriores, y que se asociaron a complicaciones relacionadas con el procedimiento $^{5-9}$.

1. Sanatorio Otamendi. Cardiología Clínica

2. Centro de Estudios en Cardiología Intervencionista (CECI).

$\triangle$ Correspondencia:DrA. Matías Rodríguez-Granillo.mrodriguezgranillo@gmail.com Los autores no declaran conflictos de intereses

Recibido: 25/09/2018|Aceptado: 24/10/2018
La nuevas generaciones de prótesis y la mayor experiencia de los equipos tratantes extendieron su uso a pacientes con menor riesgo quirúrgico, hasta llegar al año 2017, durante el cual tanto la Sociedad Europea de Cardiología y de la American Heart Association/American College of Cardiology publicaron recomendaciones acerca de la no inferioridad del TAVR vs. la cirugía convencional de reemplazo en pacientes con estenosis aórtica severa sintomática y riesgo intermedio ${ }^{9-10}$.

Solo existe una única revisión del tema en nuestro país publicada en el año 2014, en donde no se valoran las nuevas evidencias ${ }^{11}$. No existen otras revisiones sistemáticas en castellano y es el objetivo de este manuscrito.

\section{MATERIAL Y MÉTODOS}

\section{Criterios de búsqueda bibliográfica y selección de artículos}

Se realizó una búsqueda bibliográfica de todos los Ensayos Clínicos Randomizados (ECR) controlados acerca de TAVR en pacientes con riesgo quirúrgico intermedio hasta agosto de 2017. La búsqueda se realizó en inglés, pero no hubo restricciones en cuanto al idioma de la publicación original. La revisión sistemática se realizó en PubMed, Embase, Cochrane Central Register of Controlled Trials y en Scopus el 

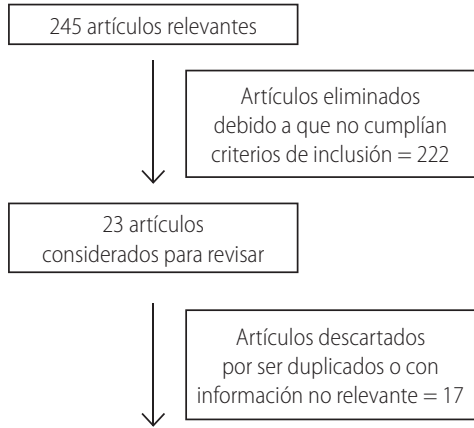

$$
\begin{aligned}
& 5 \text { ensayos clínicos rando- } \\
& \text { mizados con un total de } \\
& 4765 \text { pacientes a analizar }
\end{aligned}
$$

Figura 1. Diagrama de flujo de la búsqueda bibliográfica realizada para esta monografía.

día 14 de agosto de 2017. Se utilizaron como palabras clave: "Aortic valve replacement"; "TAVR", "TAVI”, "SAVR” e "intermediate risk". Adicionalmente se buscaron manualmente citas y referencias en castellano con los mismos criterios de búsqueda en Google Scholar y Scielo a fin de encontrar y conocer lo escrito en nuestro idioma en otros motores de búsqueda científicos para incorporar, si cumpliesen con lo antes mencionado, esa información a esta revisión. Como criterio de inclusión para su evaluación se seleccionaron a todos los artículos que informaran un riesgo quirúrgico por score STS-PROM $\geq 4 \%$ o EuroSCORE II $\geq 8-10 \%$ o aquellas en donde se pudiera discriminar con claridad a los pacientes de riesgo intermedio. Además se tuvieron en cuenta todas aquellas publicaciones que incluyeron datos acerca de la seguridad y eficacia entre TAVR y RVAO.

\section{Medidas de evaluación}

La medida de eficacia clínica fue la mortalidad global, pudiéndose dividir esta, si hubiera estado informada, en temprana (en el hospital o a 30 días) o tardía (luego de los 30 días), de acuerdo a como fue identificada en cada ECR seleccionado. Además se valoraron los resultados de los eventos duros tales como infarto de miocardio (IAM), accidente cerebrovascular (ACV) o isquémico transitorio (AIT), nuevas hospitalizaciones por insuficiencia cardíaca, requerimiento de reintervención, necesidad de implante de marcapaso definitivo, falla renal, sangrado mayor, insuficiencia aórtica, tiempo de internación y otras complicaciones del procedimiento para evaluar, además de la eficacia clínica, la seguridad. Por último, se analizaron valores ecocardiográficos de gradientes de presión, área valvular aórtica cuando hubiere estado informada e insuficiencia paravalvular.

\section{Presentación de resultados}

En la sección de resultados se presentan los datos de cada ECR en particular y un análisis de los resultados más relevantes. En la sección Discusión se evaluaron las fortalezas y debilidades de los ECR revisados y comparaciones entre cada uno de ellos.

\section{Análisis estadístico}

Se tomó el punto final primario de muerte global en el hospital y a 30 días, al año y a los dos años de seguimiento comparando toda la información publicada acerca de los ECR evaluados y la suma de sus resultados. Se utilizó chi cuadrado para evaluar variables categóricas y odds ratio para conocer la posibilidad de la ocurrencia del evento en cada uno de los ensayos clínicos seleccionados y en los resultados a dos años de aquellos que informaron esos datos en sus manuscritos. Los resultados se presentan en la sección correspondiente y en tablas y figuras. El análisis estadístico se realizó con el programa SPSS V.17.1.

\section{RESULTADOS}

\section{Estudios aleatorizados}

Se analizaron 245 artículos potencialmente relevantes. Una vez que se removieron artículos duplicados y aquellos que no cumplían con los criterios de inclusión, fueron finalmente 5 los ECR evaluados (Figura 1). Uno de ellos, el estudio STACCATO, fue incorporado a pesar de haber sido terminado tempranamente ${ }^{12}$. De los ECR analizados, dos se realizaron en Norteamérica, dos en Europa y uno en ambos continentes, siendo los seleccionados: "A prospective, randomized trial of transapical transcatheter aortic valve implantation vs. surgical aortic valve replacement in operable elderly patients with aortic stenosis: the STACCATO trial"12; "Transcatheter Versus Surgical Aortic Valve Replacement in Patients With Severe Aortic Valve Stenosis 1-Year Results From the All-Comers NOTION Randomized Clinical Trial"13; "Transcatheter aortic-valve replacement with a self expanding prosthesis. US Pivotal" 14; "Transcatheter or Surgical Aortic-Valve Replacement in Intermediate-Risk Patients. PARTNER $2 A$ "15 y "Surgical or Transcatheter Aortic-Valve Replacement in Intermediate-Risk Patients"16. Un estudio utilizó solo acceso transapical ${ }^{12}$; dos de ellos el acceso transfemoral preferentemente ${ }^{13,16} \mathrm{y}$ dos utilizaron ambos accesos pero con una randomización estratificada de acuerdo a la accesibilidad del paciente ${ }^{14,15}$. Se analizaron en total 4765 pacientes con estenosis aórtica severa sintomática de riesgo intermedio, 2445 en el grupo TAVR y 2388 pacientes en el grupo RVAO. Las características de cada uno de los ECR analizados, junto a las características clínicas previas más relevantes, el acceso vascular utilizado para realizar la TAVR y la prótesis utilizada se presentan en la Tabla 1.

El primero de ellos fue el STACCATO trial, un pequeño estudio danés realizado por Hans Nielsen et al. y publicado en 2012 en la revista Eurointervention. Tuvo como objetivo primario el punto final combinado compuesto de mortalidad de cualquier causa, ACV y falla renal con requerimiento de diálisis a 30 días, comparando al RVAO con TAVR realizada por acceso transapical con una prótesis Edwards SAPIEN balón expandible ${ }^{12}$. Se excluyeron pacientes con enfermedad coronaria que pudiera ser tratada tanto con intervención percutánea coronaria (ICP) como con cirugía de revascularización miocárdica (CRM), IAM previo, ICP en los últimos doce meses, cirugía cardíaca previa, cirugía de urgencia (dentro de las 24 horas), condición cardíaca inestable (con requerimientos de inotrópicos o dispositivos de asistencia mecánica en las últimas 24 hs.), infecciones concomitantes con requerimiento de antibióticos, ACV dentro del último mes, función respiratoria reducida, falla renal tratada con hemodiálisis, alergia a la aspirina, clopidogrel, prasugrel o al contraste. Si bien el STACCATO fue el primer estudio randomizado en comparar el acceso transapical en pacientes con riesgo quirúrgico intermedio (mortalidad a 30 días), fue terminado tempranamente ( $1 / 3$ del reclutamiento planificado) 
TABLA 1. Características de los estudios clínicos randomizados incluidos en el análisis.

\begin{tabular}{|c|c|c|c|c|c|c|c|c|c|c|c|}
\hline & & & & & \multicolumn{7}{|c|}{ Características clínicas previas \% } \\
\hline Trial & $\begin{array}{c}\text { Número de } \\
\text { pacientes. }\end{array}$ & $\begin{array}{c}\text { Seguimiento } \\
\text { (meses) }\end{array}$ & $\begin{array}{l}\text { Tipo de } \\
\text { válvula }\end{array}$ & $\begin{array}{c}\text { Acceso vascular } \\
\text { femoral } \%\end{array}$ & $\begin{array}{l}\text { Edad (DE) } \\
\text { (años) }\end{array}$ & $\begin{array}{l}\text { Riesgo } \\
\text { medio }\end{array}$ & Mujeres & DM & IR & EPOC & FA \\
\hline STACCATO (1) & 72 & 3 & $\begin{array}{l}\text { Edwards } \\
\text { SAPIEN }\end{array}$ & 0 & $81(4)$ & $3,3(1,4)$ & 70 & 5,7 & NR & 2,9 & NR \\
\hline NOTION (2) & 280 & 24 & CoreValve & 96,5 & $79,1(14,8)$ & $3,0(1,6)$ & 46,8 & 19,3 & 1,1 & 11,8 & 26,7 \\
\hline US Pivotal (3) & 795 & 36 & CoreValve & Estrato & $83,4(6,7)$ & $7,4(3,1)$ & 46,8 & 38,7 & 12,7 & 11,1 & 44,3 \\
\hline Partner 2A (4) & 2032 & 24 & $\begin{array}{l}\text { Edwards } \\
\text { SAPIEN XT }\end{array}$ & 76,3 & $81,6(6,7)$ & $5,8(2,0)$ & 45,5 & 35,9 & 5,1 & 30,9 & 33,0 \\
\hline SURTAVI (5) & 864 & 24 & $\begin{array}{l}\text { CoreValve } \\
\text { y Evolut R }\end{array}$ & $N D^{*}$ & $79,9(6,2)$ & $4,4(1,5)$ & 43,4 & 34,1 & 1,6 & ND & 28,1 \\
\hline
\end{tabular}

DE: desvío estándar. DM: diabetes. IR: insuficiencia renal. EPOC: enfermedad pulmonar obstructiva crónica. FA: fibrilación auricular. NR: no reportado.

(1) Nielsen HH, Klaaborg KE, Nissen H, Terp K, Mortensen PE, Kjeldsen BJ et al. A prospective, randomized trial of transapical transcatheter aortic valve implantation vs. surgical aortic valve replacement in operable elderly patients with aortic stenosis: the STACCATO trial. Eurolntervention 2012;8:383-9.

(2) Thyregod HGH, Steinbrüchel DA, Ihlemann N, Nissen H, Kjeldsen BJ, Petursson P et al. Transcatheter Versus Surgical Aortic Valve Replacement in Patients With Severe Aortic Valve Stenosis 1-Year Results From the All-Comers NOTION Randomized Clinical Trial. J Am Coll Cardiol 2015;65:2184-94.

(3) Adams DH, Popma JJ, Reardon MJ, Yakubov SJ, Coselli JS, Deeb GM et al. U.S. CoreValve Clinical Investigators. Transcatheter aortic-valve replacement with a self expanding prosthesis. NEngl J Med 2014;370:1790-8.

(4) Leon MB, Smith CR, Mack MJ, Makkar RR, Svensson LG, Kodali SK et al. PARTNER 2 Investigators. Transcatheter or Surgical Aortic-Valve Replacement in Intermediate-Risk Patients. NEngl J Med 2016;374:1609-20.

(5) Reardon MJ, Van Mieghem NM, Popma JJ, Kleiman NS, Søndergaard L, Mumtaz M et al. for the SURTAVI Investigators. Surgical or Transcatheter Aortic-Valve Replacement in Intermediate-Risk Patients. N Engl J Med. 2016 Apr 28;374(17):1609-20.

\begin{tabular}{|c|c|c|c|c|c|}
\hline Grupo & Edad & Sexo & Evento adverso & Tiempo del evento & Resultado a 3 meses \\
\hline TAVR & 80 & Fem & Muerte & Preprocedimiento & No relacionado con tratamiento \\
\hline TAVR & 70 & Fem & Obstrucción TCI & Periprocedimiento & Muerte al día 1 \\
\hline TAVR & 79 & Fem & $\mathrm{ACV}$ & Día 27 & Muerte al día 36 \\
\hline TAVR & 86 & Fem & $\mathrm{ACV}$ & Día 16 & Secuela severa. Rankin score 5 \\
\hline TAVR & 81 & Fem & AIT-ACV & Periprocedimiento & Recuperación. Rankin score 3 \\
\hline TAVR & 76 & Fem & Diálisis (IRA) & Día 8 & Hemodiálisis permanente \\
\hline TAVR & 80 & Masc & Oclusión TCl & Periprocedimiento & RVAO. Recuperación \\
\hline TAVR & 79 & Masc & Ruptura aórtica & Periprocedimiento & Cirugía. Recuperación \\
\hline TAVR & 75 & Masc & Leak perivalvular & Periprocedimiento & RVAO. Recuperación \\
\hline TAVR & 82 & Fem & Leak perivalvular & Periprocedimiento & RVAO. Recuperación \\
\hline TAVR & 78 & Masc & Embolización & Periprocedimiento & Implante TAVR. Recuperación \\
\hline TAVR & 80 & Fem & ICC & Periprocedimiento & RVAO. Recuperación \\
\hline TAVR & 86 & Fem & Sangrado & Posprocedimiento & Recuperación \\
\hline RVAO & 81 & Fem & ACV & Periprocedimiento & Secuela severa. Rankin score 5 \\
\hline RVAO & 83 & Fem & Trauma torácico & Periprocedimiento & TAVR. Recuperación \\
\hline RVAO & 82 & Masc & Sangrado & Posprocedimiento & Recuperación \\
\hline
\end{tabular}

TAVR: reemplazo valvular aórtico transapical. RVAO: reemplazo valvular aórtico quirúrgico. TCl: tronco coronario izquierdo. ACV: accidente cerebrovascular. AlT: accidente isquémico transitorio. IRA: insuficiencia renal aguda. ICC: insuficiencia cardiaca congestiva.

(1) Nielsen HH, Klaaborg KE, Nissen H, Terp K, Mortensen PE, Kjeldsen BJ et al. A prospective, randomized trial of transapical transcatheter aortic valve implantation vs. surgical aortic valve replacement in operable elderly patients with aortic stenosis: the STACCATO trial. Eurolntervention 2012;8:383-9.

debido al aumento en la incidencia de eventos adversos en el grupo TAVR, tales como ruptura aórtica, embolización de prótesis, oclusión del tronco coronario izquierdo, stroke y leak perivalvular (Tabla 2).

El NOTION trial, el segundo de los ECR, se publicó en el año 2015 en el Journal of the American College of Cardiology y consistió en un análisis de superioridad, comparando una prótesis autoexpandible contra el RVAO convencional en pacientes con riesgo quirúrgico intermedio o bajo ${ }^{13}$. El sitio de acceso preferente fue el femoral, siendo el subclavio izquierdo la segunda opción. Los criterios de inclusión y exclusión fueron similares a los del ECR previo, y la randomización se estratificó de acuerdo al centro participante, edad y antecedentes de enfermedad coronaria de los pacientes.

El objetivo primario fue el punto final combinado compuesto de muerte por cualquier causa, ACV, IAM al $1^{\circ}$ y $5^{\circ}$ año luego del procedimiento basal. Los puntos finales secundarios fueron el análisis individual de cada uno de los componentes del punto final primario. También se evaluó la incidencia de muerte cardiovascular, reintervención, shock cardiogénico, endocarditis, anormalidades de la conducción cardíaca con requerimiento de implante de marcapaso permanente, fibrilación o aleteo auricular y sangrado desde el mes 1 al mes 12 de seguimiento. Dentro de los objetivos secundarios del NOTION trial se compararon parámetros hemodinámicos por ecocardiografía tales como el orificio valvular efectivo, el gradiente medio de presión y el grado de reflujo aórtico de acuerdo a las definiciones propuestas por el Consorcio Académico de Investigación Valvular - 2. ${ }^{17}$ Este fue un estudio de superioridad en donde los autores estimaron una ocurrencia del evento primario de 5\% en TAVR y del $15 \%$ en RVAO, por lo que incorporaron 142 pacientes en el grupo TAVR y 134 en el grupo RVAO, calculando un error alfa de $5 \%$ y un poder de la muestra del $80 \%$.

El análisis del punto final combinado no demostró diferencias entre ambos grupos $(13,1 \%$ vs. $16,3 \%$ para TAVR y RVAO, respectivamente, $-3,2 \%$ de diferencia absoluta; $\mathrm{p}=$ 0,43 para superioridad). En la Tabla 3 se presentan los puntos finales secundarios con diferencias estadísticamente significativas durante la hospitalización a favor de TAVR. A un año de seguimiento, la necesidad de implante de marcapaso definitivo fue significativamente mayor en el grupo TAVR, 
TABLA 3. Resultados del NOTION trial (1) durante la internación, a los 30 días y al año de seguimiento.

\begin{tabular}{|c|c|c|c|c|c|c|}
\hline & \multicolumn{3}{|c|}{ Internación o 30 días } & \multicolumn{3}{|c|}{1 año de seguimiento } \\
\hline & TAVR $\%$ & RVAO \% & Valor de $\mathrm{p}$ & TAVR \% & RVAO \% & Valor de $\mathrm{p}$ \\
\hline Sangrado mayor & 11,3 & 20,9 & 0,03 & & & \\
\hline Shock cardiogénico & 4,2 & 10,4 & 0,05 & & & \\
\hline Complicaciones vasculares & 5,6 & 1,5 & 0,1 & & & \\
\hline IRA & 0,7 & 6,7 & 0,01 & & & \\
\hline Muerte global & 2,1 & 3,7 & 0,43 & 4,9 & 7,5 & 0,38 \\
\hline Muerte cardiovascular & 2,1 & 3,7 & 0,43 & 4,3 & 7,5 & 0,25 \\
\hline ACV & 1,4 & 3,0 & 0,37 & 2,9 & 4,6 & 0,44 \\
\hline IAM & 2,8 & 6,0 & 0,20 & 3,5 & 6,0 & 0,33 \\
\hline MCP permanente & 34,1 & 1,6 & $<0,001$ & 38,0 & 2,4 & $<0,001$ \\
\hline Nueva FA o empeoramiento & 16,9 & 57,8 & $<0,001$ & 21,2 & 59,4 & $<0,001$ \\
\hline
\end{tabular}

TAVR: reemplazo valvular aórtico percutáneo. RVAO: reemplazo valvular aórtico quirúrgico. IRA: insuficiencia renal aguda. ACV: accidente cerebrovascular. IAM: infarto agudo de miocardio. MCP: marcapasos. FA: fibrilación auricular.

(1) Thyregod HGH, Steinbrüchel DA, Ihlemann N, Nissen H, Kjeldsen BJ, Petursson P et al. Transcatheter Versus Surgical Aortic Valve Replacement in Patients With Severe Aortic Valve Stenosis 1-Year Results From the All-Comers NOTION Randomized Clinical Trial. J Am Coll Cardiol 2015;65:2184-94.

TABLA 4. Complicaciones relacionadas al procedimiento en el estudio US PIVOTAL (1)

\begin{tabular}{|c|c|c|c|c|c|c|}
\hline & \multicolumn{3}{|c|}{30 días } & \multicolumn{3}{|c|}{1 año } \\
\hline & TAVR & RVAO & Valor de $p$ & TAVR & RVAO & Valor de $p$ \\
\hline Complicaciones vasculares mayores & 5,9 & 1,7 & 0,003 & 6,2 & 2,0 & 0,004 \\
\hline Sangrado fatal & 13,6 & 35,0 & $<0,001$ & 16,6 & 38,4 & $<0,001$ \\
\hline Sangrado mayor & 28,1 & 34,5 & 0,05 & 29,5 & 36,7 & 0,03 \\
\hline Falla renal aguda & 6,0 & 15,1 & $<0,001$ & 6,0 & 15,1 & $<0,001$ \\
\hline Shock cardiogénico & 2,3 & 3,1 & 0,51 & 2,3 & 3,1 & 0,51 \\
\hline Perforación cardíaca & 1,3 & 0,0 & 0,03 & 1,3 & 0,0 & 0,03 \\
\hline Implante de MCP & 19,8 & 7,1 & $<0,001$ & 22,3 & 11,3 & $<0,001$ \\
\hline Nueva FA o empeoramiento & 11,7 & 30,5 & $<0,001$ & 15,9 & 32,7 & $<0,001$ \\
\hline
\end{tabular}

TAVR: reemplazo valvular aórtico percutáneo. RVAO: reemplazo valvular aórtico quirúrgico. Sangrado fatal: intracraneal, intraespinal, intraocular o pericárdico con requerimiento de pericardiocentesis o sangrado intramuscular que causare síndrome compartimental o sangrado que causare shock hipovolémico o severa hipotensión con requerimiento de transfusión de 4 o más unidades de glóbulos rojos (UGR). Sangrado mayor: asociado a requerimiento de 2 o 3 UGR o sangrados que no cumplan con los criterios de sangrado fatal. MCP: marcapasos. FA: fibrilación auricular

(1) Adams DH, Popma JJ, Reardon MJ, Yakubov SJ, Coselli JS, Deeb GM et al. U.S. CoreValve Clinical Investigators. Transcatheter aortic-valve replacement with a self expanding prosthesis. NEngl J Med 2014;370:1790-8.

mientras que la incidencia de nueva fibrilación auricular o empeoramiento de la misma fue significativamente mayor en el grupo RVAO (Tabla 3).

En cuanto a las diferencias ecocardiográficas, el orificio valvular efectivo tuvo una mejora significativamente mayor en el grupo TAVR; sin embargo, el grado de regurgitación aórtica fue significativamente menor en el grupo RVAO $(\mathrm{p}<0,001)$, tanto a los 3 meses como al año de seguimiento.

El ECR US Pivotal ${ }^{14}$, publicado en 2014 y que utilizó CoreValve, una válvula de nitinol autoexpandible, se realizó en 34 centros de los Estados Unidos y tuvo como objetivo comparar el tratamiento de pacientes con riesgo quirúrgico aumentado que iban a someterse al reemplazo valvular por TAVR o por RVAO. Definieron como riesgo aumentado a aquellos pacientes en los que dos cirujanos y un cardiólogo intervencionista estimaron un riesgo de muerte a 30 días luego de la cirugía del $15 \%$ o más o con riesgo de complicaciones irreversibles a 30 días de, por lo menos, 50\%, utilizando el STS-PROM como score de riesgo quirúrgico. Este score provee un riesgo estimado de muerte a 30 días en pacientes a los que se les realizará un RVAO en base a variables demográficas y de procedimiento ${ }^{18}$. La vía transfemoral fue la de elección salvo que por tomografía computada hubiera algún problema con el sitio de acceso, siendo opciones la subclavia o aórtica directa. Para evitar sesgos se realizó una randomización estratificada dividida de acuerdo a la vía de acceso, 1:1. El seguimiento fue al primero y sexto mes y luego al año. El objetivo primario fue muerte de cualquier causa. Como puntos secundarios el evento combinado de muerte de cualquier causa, infarto, $\mathrm{ACV}$ o reintervención a 30 días y al año, además de cada uno de ellos de forma individual. Este ECR tuvo la particularidad que el objetivo primario fue de no inferioridad, con una margen de 7,5; un error alfa de 0,05 y un poder del $80 \%$, pero además se preespecificó una segunda hipótesis de superioridad que evaluó la incidencia de eventos mayores cardiovasculares y cerebrales a 30 días (MACCE). El STS-PROM estimado del estudio en promedio fue de $7,4 \%$ de mortalidad a los 30 días. Además se tuvo en cuenta la fragilidad de los pacientes, demostrando que los pacientes seleccionados tenían un score alto; el 55,8\% tenían severas comorbilidades.

Los resultados de este trabajo demostraron la no inferioridad de TAVR con respecto al objetivo primario, con una reducción de riesgo absoluta del 4,9\% ( $\mathrm{p}<0,001$ para no inferioridad y $\mathrm{p}=0,04$ para superioridad); al comparar el objetivo primario entre diferentes subgrupos solo se encontraron diferencias significativas a favor del TAVR en las mujeres (hazard ratio $[\mathrm{HR}]=0,56$; IC95\%: 0,33-0,95). En pacientes donde se implantó exitosamente la válvula, TAVR no fue inferior que RVAO al comparar los valores basales y a un año de seguimiento entre gradiente medio valvular basal $(-39,04 \mathrm{mmHg} v$ s. $-35,42 \mathrm{mmHg} ; \mathrm{p}<0,001$ para no inferioridad) y en área valvular $\left(1,20 \mathrm{~cm}^{2}\right.$ vs. $0,81 \mathrm{~cm}^{2} ; \mathrm{p}<0,001$ para no inferioridad). Las complicaciones a 30 días se muestran en la Tabla 4, en donde se evidencia que el grupo TAVR tuvo significativamente mayor incidencia de complicaciones vasculares mayores, perforación cardíaca e implante de marcapaso, y el grupo RVAO significativamente mayor inciden- 
TABLA 5. Complicaciones periprocedimiento y a 30 días de los diferentes ECR

\begin{tabular}{|l|c|c|c|c|c|c|c|c|c|c|c|c|c|c|c|}
\hline & \multicolumn{3}{|c|}{ STACCATO (1) } & \multicolumn{3}{|c|}{ NOTION (2) } & \multicolumn{3}{c|}{ US PIVOTAL (3) } & \multicolumn{3}{c|}{ PARTNER 2A (4) } & \multicolumn{3}{c|}{ SURTAVI (5) } \\
\hline & TAVR & RVAO & $\mathrm{p}$ & TAVR & RVAO & $\mathrm{p}$ & TAVR & RVAO & $\mathrm{p}$ & TAVR & RVAO & $\mathrm{p}$ & TAVR & RVAO & IC 95\% \\
\hline Muerte & 8,3 & 0,0 & 0,1 & 2,1 & 3,7 & 0,4 & 3,3 & 4,5 & 0,6 & 6,1 & 8,0 & 0,11 & 2,2 & 1,7 & $-2,2 \mathrm{a} 1,8$ \\
\hline IAM & 2,7 & 0 & 0,8 & 2,8 & 6,0 & 0,2 & ND & ND & - & 1,2 & 1,9 & 0,2 & 0,9 & 1,0 & $-1,0 \mathrm{a} 0,9$ \\
\hline IRA & 2,7 & 0 & 0,8 & 0,7 & 6,7 & 0,01 & 6,0 & 15,1 & $<0,001$ & 1,3 & 3,1 & 0,006 & 1,7 & 4,4 & $-4,4 \mathrm{a}-1$ \\
\hline ACV/AIT & 8,3 & 2,8 & 0,2 & 2,8 & 3,0 & 0,94 & 4,9 & 6,2 & 0,4 & 5,5 & 6,1 & 0,57 & 3,4 & 5,6 & $-4,2 \mathrm{a} 0,2$ \\
\hline CVM & 8,3 & 2,8 & 0,2 & 5,6 & 1,5 & 0,1 & 5,9 & 1,7 & 0,003 & 7,9 & 5,0 & 0,008 & 6,0 & 1,1 & $3,2 \mathrm{a} 6,7$ \\
\hline FA & ND & ND & - & 16,9 & 57,8 & $<0,001$ & 11,7 & 30,5 & $<0,001$ & 9,1 & 26,4 & $<0,001$ & 12,9 & 43,4 & $-34 \mathrm{a}-26$ \\
\hline MCP & ND & ND & - & 34,1 & 1,6 & $<0,001$ & 19,8 & 7,1 & $<0,001$ & 8,5 & 6,9 & 0,17 & 25,9 & 6,6 & $16 \mathrm{a} 22$ \\
\hline SS & 2,7 & 3 & 0,8 & 11,3 & 20,9 & 0,03 & 13,6 & 35,0 & $<0,001$ & 10,4 & 43,4 & $<0,001$ & 12,2 & 9,3 & $-0,1 \mathrm{a} 6$ \\
\hline
\end{tabular}

CVM: complicación vascular mayor. SS: sangrado severo. IAM: Infarto agudo de miocardio; IRA: Insuficiencia renal aguda; ACV/AIT: Accidente cerebrovascular/Accidente isquémico transitorio; FA: Fibrilación auricular; MCP: Marcapaso

(1) Nielsen HH, Klaaborg KE, Nissen H, Terp K, Mortensen PE, Kjeldsen BJ et al. A prospective, randomized trial of transapical transcatheter aortic valve implantation vs. surgical aortic valve replacement in operable elderly patients with aortic stenosis: the STACCATO trial. Eurolntervention 2012;8:383-9.

(2) Thyregod HGH, Steinbrüchel DA, Ihlemann N, Nissen H, Kjeldsen BJ, Petursson P et al. Transcatheter Versus Surgical Aortic Valve Replacement in Patients With Severe Aortic Valve Stenosis 1-Year Results From the All-Comers NOTION Randomized Clinical Trial. J Am Coll Cardiol 2015;65:2184-94.

(3) Adams DH, Popma JJ, Reardon MJ, Yakubov SJ, Coselli JS, Deeb GM et al. U.S. CoreValve Clinical Investigators. Transcatheter aortic-valve replacement with a self expanding prosthesis. N Engl J Med 2014;370:1790-8.

(4) Leon MB, Smith CR, Mack MJ, Makkar RR, Svensson LG, Kodali SK et al. PARTNER 2 Investigators. Transcatheter or Surgical Aortic-Valve Replacement in Intermediate-Risk Patients. NEngl J Med 2016:374:1609-20.

(5) Reardon MJ, Van Mieghem NM, Popma JJ, Kleiman NS, Søndergaard L, Mumtaz M et al. for the SURTAVI Investigators. Surgical or Transcatheter Aortic-Valve Replacement in Intermediate-Risk Patients. N Engl J Med. 2016 Apr 28;374(17):1609-20.

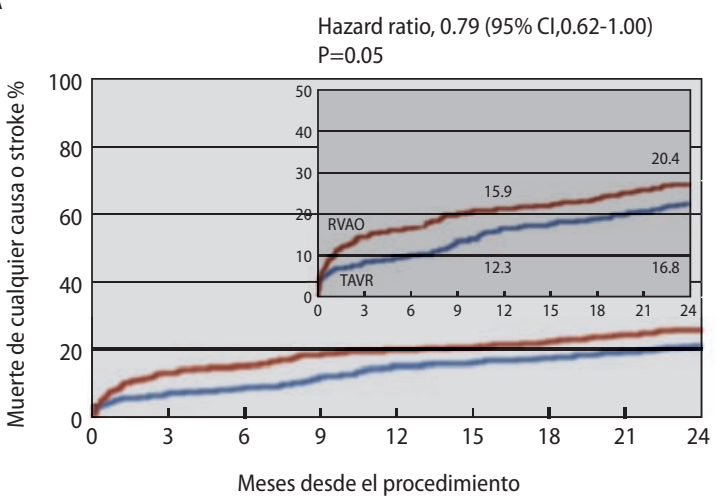

No en riesgo

$\begin{array}{lllllllllll}\text { TAVR } & 775 & 718 & 709 & 685 & 663 & 652 & 644 & 634 & 612 & \text { TAVR }\end{array}$

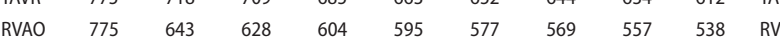

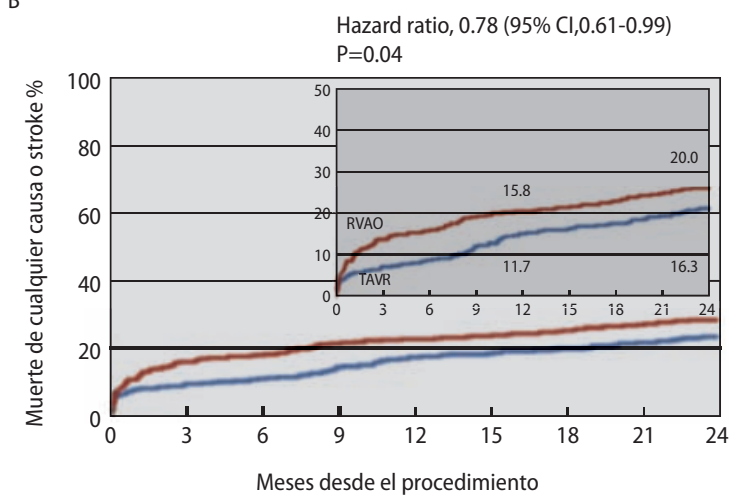

No en riesgo

$\begin{array}{llllllllll}\text { TAVR } & 762 & 717 & 708 & 685 & 663 & 652 & 644 & 634 & 612\end{array}$

Figura 2. Análisis por subgrupos del estudio PARTNER 2 cohorte A. Análisis por subgrupo de vía transfemoral, incidencia de muerte de cualquier causa o stroke por intención de tratar (A) y tratamiento efectivo (B), comparación entre RVAO y TAVR con una diferencia a favor del grupo TAVR. Leon MB, Smith CR, Mack MJ, Makkar RR, Svensson LG, Kodali SK et al. PARTNER 2 Investigators. Transcatheter or Surgical Aortic-Valve Replacement in Intermediate-Risk Patients. N Engl J Med 2016;374:1609-20

cia de sangrado fatal y sangrado mayor, falla renal aguda y nueva fibrilación auricular. A 1 año se mantuvieron estas diferencias a favor del grupo TAVR, que como contrapartida requirió uso de marcapaso definitivo en casi 1 de cada 5 pacientes tratados (Tabla 4).

El estudio PARTNER 2 cohorte A fue publicado en el New England Journal of Medicine en abril del año 2016, y comparó al RVAO con TAVR con un dispositivo de segunda generación en pacientes con estenosis aórtica severa y un perfil de riesgo quirúrgico intermedio, realizado en 57 centros de Canadá y Estados Unidos entre los años 2011 y $2013^{15}$. Para incluir a un paciente en el estudio un equipo multidisciplinario evaluó el riesgo quirúrgico de acuerdo al score STS, incorporando a todos aquellos que tuvieron un riesgo entre 4 y $8 \%$, con la salvedad de que pacientes con riesgo $<4 \%$ pudieron ser incluidos cuando tuvieran condiciones preexistentes que no estuvieran representadas en el modelo de riesgo del STS ${ }^{19}$. Después de cumplir todos los criterios de inclusión se evaluó el sitio de acceso de cada paciente para separar a los que podían ser abordados por vía femoral de aquellos a los que debían realizarle el procedimiento por vía torácica, para luego estratificar las cohortes de acuerdo al sitio de acceso (transfermoral o transtorácico), siendo asignados aleatoriamente (1:1) al grupo RVAO o TAVR.

El objetivo final primario del ECR fue comparar los eventos combinados de muerte de cualquier causa o stroke incapacitante a dos años, analizándose los resultados teniendo en cuenta la intención de tratar. Se definió como stroke incapacitante a aquel con escala de Rankin de por lo menos 2 a los 90 días del evento clínico (0 si no poseía sintomatología, 6 si hubiera fallecido) $)^{17}$, evaluados por un neurólogo que fue ciego al grupo de tratamiento implementado. Este fue un estudio de no inferioridad donde los autores asumieron que a dos años de seguimiento iba a haber 30\% de eventos en cada uno de los grupos, por lo que estimaron una muestra de 2000 pacientes, con un poder del $80 \%$ un alfa de 0,025 y un RR para no inferioridad de 1,2 . Además se realizó un análisis de regresión logística multivariable para conocer asociaciones entre complicaciones durante el procedimiento y mortalidad. En total se randomizaron 2032 pacientes, de los 
TABLA 6. Comparación entre todos los ECR entre TAVR y RVAO a 30 días, 1 y 2 años de seguimiento y resultados globales de los ECR con seguimiento a dos años en cuanto a muerte de cualquier causa.

\begin{tabular}{|c|c|c|c|c|}
\hline & $\operatorname{TAVR}(\mathrm{n})$ & RVAO (n) & OR (IC 95\%) & $p$ \\
\hline \multicolumn{5}{|c|}{ Resultados hospitalarios y a 30 días de seguimiento } \\
\hline STACATTO & $8,3 \%(2 / 34)$ & $0,0 \%(0 / 36)$ & $2,25(0,19-26)$ & 0,3 \\
\hline NOTION & $2,1 \%(3 / 142)$ & $3,7 \%(5 / 134)$ & $0,55(0,17-2,37)$ & 0,6 \\
\hline US PIVOTAL & $3,3 \%(13 / 394)$ & $4,5 \%(18 / 401)$ & $0,72(0,35-1,5)$ & 0,49 \\
\hline PARTNER 2 A & $3,9 \%(39 / 1011)$ & $4,1 \%(41 / 1021)$ & $0,95(0,61-1,5)$ & 0,9 \\
\hline SURTAVI & $2,2(19 / 864)$ & $1,7(13 / 796)$ & $1,35(0,66-2,76)$ & 0,40 \\
\hline \multicolumn{5}{|c|}{1 año de seguimiento } \\
\hline NOTION & $4,9 \%(7 / 142)$ & $7,5 \%(10 / 134)$ & $0,64(0,23-1,74)$ & 0,53 \\
\hline US PIVOTAL & $14,2 \%(56 / 394)$ & $19,1 \%(77 / 401)$ & $0,69(0,47-1,01)$ & 0,07 \\
\hline PARTNER 2 A & $12,3 \%(123 / 1011)$ & $12,9 \%(124 / 1021)$ & $1,02(0,76-1,30)$ & 0,9 \\
\hline SURTAVI & $6,7 \%(58 / 864)$ & $6,8 \%(54 / 796)$ & $0,98(0,77-1,24)$ & 0,9 \\
\hline \multicolumn{5}{|c|}{2 años de seguimiento } \\
\hline NOTION & $8,0 \%(11 / 142)$ & $9,8 \%(13 / 134)$ & $0,78(0,33-1,81)$ & 0,71 \\
\hline PARTNER $2 \mathrm{~A}$ & $16,7 \%(166 / 1011)$ & $18.0 \%(170 / 1021)$ & $0,98(0,77-1,24)$ & 0,9 \\
\hline SURTAVI & $11,4 \%(98 / 864)$ & $11,6 \%(92 / 796)$ & $0,97(0,72-1,32)$ & 0,9 \\
\hline Global a 2 años & $13,6 \%(275 / 2017)$ & $14,1 \%(275 / 1951)$ & $0,96(0,80-1,15)$ & 0,9 \\
\hline
\end{tabular}

1. Nielsen HH, Klaaborg KE, Nissen H, Terp K, Mortensen PE, Kjeldsen BJ et al. A prospective, randomized trial of transapical transcatheter aortic valve implantation vs. surgical aortic valve replacement in operable elderly patients with aortic stenosis: the STACCATO trial. Eurolntervention 2012;8:383-9.

2. Thyregod HGH, Steinbrüchel DA, Ihlemann N, Nissen H, Kjeldsen BJ, Petursson P et al. Transcatheter Versus Surgical Aortic Valve Replacement in Patients With Severe Aortic Valve Stenosis 1-Year Results From the All-Comers NOTION Randomized Clinical Trial. J Am Coll Cardiol 2015;65:2184

3. Adams DH, Popma JJ, Reardon MJ, Yakubov SJ, Coselli JS, Deeb GM et al. U.S. CoreValve Clinical Investigators. Transcatheter aortic-valve replacement with a self expanding prosthesis. NEngl J Med 2014;370:1790-8.

4. Leon MB, Smith CR, Mack MJ, Makkar RR, Svensson LG, Kodali SK et al. PARTNER 2 Investigators. Transcatheter or Surgical Aortic-Valve Replacement in Intermediate-Risk Patients. NEngl J Med 2016;374:1609-20

5. Reardon MJ, Van Mieghem NM, Popma JJ, Kleiman NS, Søndergaard L, Mumtaz M et al. for the SURTAVI Investigators. Surgical or Transcatheter Aortic-Valve Replacement in Intermediate-Risk Patients. N Engl J Med. 2016 Apr 28;374(17):1609-20

cuales 1011 fueron asignados a TAVR (775 transfermoral y 236 transtorácico). El STS medio fue de 5,8\%. Hubo 4,6\% de drop-out, 77 pacientes del grupo RVAO y 17 del grupo TAVR, principalmente porque luego de la randomización no quisieron realizarse el procedimiento. Como puede apreciarse en la Tabla 5, a 30 días no hubo diferencias en cuanto a muerte y stroke entre los grupos del ECR, y esos mismos resultados se mantuvieron a 2 años de seguimiento, tanto en el análisis por tratamiento ( $\mathrm{HR}=0,87$; IC95\%: 0,71-1,07; $\mathrm{p}=0,18)$ o por intención de tratar $(\mathrm{HR}=0,89$; IC95\%: 0,731,09; $p=0,25$ ) (Tabla 6), cumpliendo en ambos casos el criterio de no inferioridad ( $\mathrm{p}=0,001$ en el análisis por intención de tratar y $\mathrm{p}<0,001$ en el análisis por tratamiento). Al analizar la vía de acceso hubo una tendencia a menor ocurrencia del evento final primario en el grupo transfemoral comparado con RVAO en el análisis de intención de tratar y una diferencia significativa en el análisis por tratamiento (Figura 2). En el análisis multivariado el stroke incapacitante, sangrado severo, la injuria renal aguda y las complicaciones vasculares mayores se asociaron independientemente con mayor riesgo de muerte a los dos años de seguimiento, tanto en el grupo RVAO como en TAVR ( $p<0,001$ en todas las comparaciones). En el seguimiento alejado los puntos finales secundarios fueron significativamente menores en el grupo TAVR en sangrado severo $(17,4 \%$ vs $47,0 \%$, TAVR vs RVAO, $p<0,001)$, injuria renal aguda $(3,8 \%$ vs $6,2 \%$, TAVR vs RVAO, $\mathrm{p}=0,02)$ y nueva fibrilación auricular $(11,3 \%$ vs $27,3 \%$, TAVR vs RVAO, $\mathrm{p}<0,001)$, mientras que las complicaciones vasculares mayores fueron significativamente menores en el grupo RVAO (8,6\% vs $5,5, \mathrm{p}=0.006)$. En cuanto a los hallazgos ecocardiográficos, hubo mayor regurgitación paravalvular moderada y severa en el grupo TAVR a los 30 días (3,7\%) y esta se relacionó, a dos años de seguimiento con un aumento significativo en la incidencia de muerte, sin importar el grupo asignado (HR 2,85, IC 95\% 1,57-5,21, p<
0,001); no así cuando la insuficiencia aórtica fue leve (HR 0,95, IC 95\% 0,63-1,45; p=0.82) (Figura 3). Una explicación a este fenómeno puede deberse a que en este estudio no se utilizó la tomografía computada multicorte para evaluar correctamente las dimensiones del anillo valvular y así poder elegir un tamaño protésico adecuado ${ }^{10,20-22}$.

En el PARTNER 2 cohorte A la incidencia de stroke fue similar en ambos grupos y la revascularización coronaria fue mayor en el grupo RVAO que en el TAVR (14,5\% vs. 3,9\%), sin ser esta condición un factor deletéreo en cuanto a la mortalidad o el riesgo de stroke.

El estudio SURTAVI publicado en marzo de 2017 en el New England Journal of Medicine fue randomizado, multinacional y de no inferioridad, que evaluó una prótesis autoexpandible en pacientes con riesgo quirúrgico de muerte a 30 días entre el 3 al 15\% (score STS-PROM) ${ }^{16}$. Además, los autores tuvieron en cuenta otros parámetros como la fragilidad y las enfermedades asociadas para estadificar a los pacientes. En este estudio la evaluación del anillo valvular se realizó mediante tomografía computada multicorte. El sitio de acceso en el grupo TAVR fue preferentemente transfemoral salvo contraindicaciones. El punto final primario fue la incidencia de muerte de cualquier causa o stroke incapacitante a los 24 meses de seguimiento. La definición de stroke incapacitante siguió los criterios del Consorcio Académico de Investigación en Válvulas (VARC de sus siglas en inglés $)^{17}$. Además, se realizó un análisis de puntos secundarios preespecificados (muerte y stroke incapacitante a los 12 meses). Otros puntos secundarios fueron el combinado de muerte de cualquier causa, infarto de miocardio, stroke de cualquier tipo y cualquier reintervención. Un equipo ecocardiográfico independiente evaluó la regurgitación aórtica a 24 meses. Para el análisis estadístico se siguió el método bayesiano, determinando que el TAVR se declararía no inferior a RVAO en cuanto al objetivo primario si la proba- 


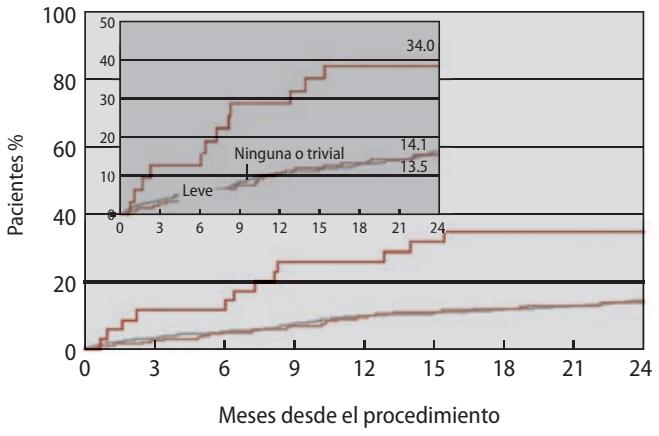

№ en riesgo

$\begin{array}{lllllllll}\text { Ninguno o trivial } 701 & 678 & 664 & 647 & 628 & 621 & 612 & 605 & 585\end{array}$ $\begin{array}{llllllllll}\text { Leve } & 210 & 204 & 199 & 194 & 188 & 184 & 182 & 180 & 175\end{array}$ $\begin{array}{lllllllll}\text { Moderada o severa } 36 & 32 & 32 & 26 & 26 & 24 & 22 & 22 & 21\end{array}$

Figura 3. Curvas de sobrevida de acuerdo al grado de regurgitación aórtica paravalvular luego del implante de TAVR en el estudio PARTNER 2 Cohorte $A$, evaluado por ecocardiograma. Comparación entre leve vs. ninguna o trivial (HR 0,95 IC 95\% 0,63-1,45, $p=0.82$ ) y entre moderada o severa vs. ninguna o trivial (HR 2,85, IC 95\%, 1,57-5,21, p<0,001), y diferencias entre la evolución de acuerdo al grado de regurgitación a dos años de seguimiento (panel mas grande). Leon MB, Smith CR, Mack MJ, Makkar RR, Svensson LG, Kodali SK et al. PARTNER 2 Investigators. Transcatheter or Surgical Aortic-Valve Replacement in Intermediate-Risk Patients. N Engl J Med 2016;374:1609-20.

bilidad posterior de no-inferioridad con un margen de 0,07 fuera más de 0,971. Se tomó un error alfa de 0,05.

Se randomizaron en total 1746 pacientes en 87 centros de Estados Unidos, Europa y Canadá entre junio de 2012 y junio de 2016, con un total de 864 pacientes en el grupo TAVR y de 796 en el grupo RVAO). La edad promedio de los pacientes fue de 79.8 años $(\mathrm{DE}=6,2)$ y el promedio de STS-PROM fue de $4,5 \pm 1,6 \%$.

De acuerdo al análisis de intención de tratar, la incidencia del objetivo final primario de muerte + stroke incapacitante a 24 meses fue del $12,6 \%$ en el grupo TAVR vs. $14,0 \%$ en el grupo TAVR, como se describe en la Figura 4 (IC95\% [análisis bayesiano] para la diferencia, $-5,2$ a 2,3\%; probabilidad posterior de no inferioridad $>0,999$ ). Hubo 71 pacientes asignados al grupo cirugía que no fueron finalmente operados. No hubo diferencias en cuanto a la mortalidad ni tampoco diferencias geográficas en los resultados. En cuanto a los eventos periprocedimiento, hubo una tendencia a favor de menor cantidad de complicaciones en el grupo TAVR, como se muestra en la Tabla 5. Los resultados ecocardiográficos mostraron que hubo mayor incidencia de insuficiencia aórtica en el grupo TAVR (5,3 vs. 0,6\%; IC95\%: 2,8-6,8\%). Los autores informaron que encontraron que el RVAO se asoció a mayor incidencia de injuria renal aguda, fibrilación auricular y requerimientos de transfusiones, mientras que TAVR tuvo mayor incidencia de regurgitación aórtica e implante de marcapaso, sin esto último relacionarse a un aumento de la mortalidad a largo plazo.

\section{DISCUSIÓN}

Una revisión sistemática identifica los trabajos de investigación relevantes mediante el uso de una estrategia de búsqueda e inclusión documentada meticulosamente, desarrollada antes del inicio de la búsqueda. Este método de revisión y síntesis de la literatura minimiza el sesgo de inclusión que puede ocurrir cuando se utiliza un enfoque narrativo. Se han analizado aquí datos de los estudios clínicos aleatorizados de pacientes con EAO candidatos a cirugía presentados tanto en Estados Unidos como en Europa ${ }^{23-26}$. Una vez que

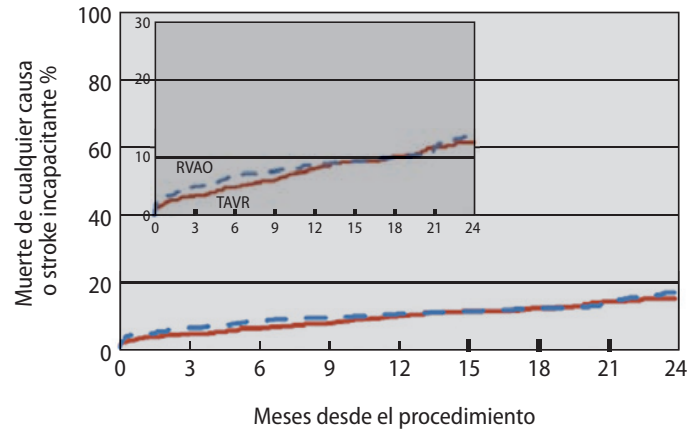

\begin{tabular}{llllll|} 
NNo en riesgo & \multicolumn{5}{l}{} \\
TAVR & 864 & 755 & 612 & 456 & 272 \\
RVAO & 796 & 674 & 555 & 407 & 241 \\
\hline \multicolumn{5}{|l|}{ Incidencia a 24 meses (\%) } \\
\hline TAVR & RVAO & IC 95\% \\
\hline 12,6 & 14,0 & $-5,2$ a 2,3 \\
\hline
\end{tabular}

Figura 4. Curvas de sobrevida libre de objetivo final primario a 24 meses de seguimiento en el estudio SURTAVI. Reardon MJ, Van Mieghem NM, Popma JJ, Kleiman NS, Søndergaard L, Mumtaz M et al. for the SURTAVI Investigators. Surgical or Transcatheter Aortic-Valve Replacement in Intermediate-Risk Patients. N Engl J Med. 2016 Apr 28;374(17):1609-20

se eliminaron artículos duplicados y aquellos que no cumplían con los criterios de inclusión, fueron 5 los ECR evaluados. Hasta la fecha existe una amplia cantidad de ensayos clínicos y metaanálisis que comparan TAVR vs. RVAO en pacientes de alto riesgo quirúrgico, que sugieren que el reemplazo percutáneo tiene un mayor beneficio en cuanto a la mortalidad. Se trató de analizar aquí si existen evidencias para prolongar la indicación a pacientes con riesgo quirúrgico estándar intermedio ${ }^{14,27-30}$.

El STACCATO trial fue un estudio pequeño randomizado que finalizó tempranamente por un aumento mayor al esperado de eventos adversos en el grupo TAVR ${ }^{12}$. Debe analizarse con cautela ya que presenta las siguientes limitaciones: primero, los investigadores eligieron una prótesis antigua, superada por las nuevas generaciones de válvulas ${ }^{14-16}$. Segundo, el acceso fue transapical, relacionado con peores resultados al compararse con la vía transfemoral, informado en numerosas series y metaanálisis ${ }^{31-35}$. Tercero, al finalizar prematuramente ( $1 / 3$ del reclutamiento planificado) no puede descartarse que los resultados obtenidos se deban al azar.

El NOTION trial tuvo como objetivo analizar el compuesto de muerte de cualquier causa, stroke e infarto a un año de seguimiento en pacientes de riesgo intermedio y bajo ${ }^{13}$. A pesar de que la mortalidad y los resultados alejados fueron similares a otras series de pacientes con las mismas características, los autores indican como una limitante la falta de experiencia de los médicos tratantes a las prótesis utilizadas.

Además, el tamaño muestral en ambos fue pequeño, y la chance no puede descartarse en los resultados del primero; en el caso del NOTION puede que esta limitación haya impedido reconocer diferencias entre ambos grupos ${ }^{13}$. Por último, hubo mejoras sustanciales en la tecnología tanto de las válvulas como del método que se utilizó en estos ECR para realizar las mediciones del anillo. En ambos se utilizó el ecocardiograma transesofágico 2-D, el gold standard en ese momento, mientras que actualmente se utiliza la tomografía multicorte, ya que el primero demostró en diferentes series una subestimación en la medición del anillo valvular causando regurgitación aórtica sistemáticamente ${ }^{20-22}$. A pesar de ello, el valor de estos ECR es fundamental, porque en 
ellos se vislumbra que los abordajes que no sean transfemorales a la hora de elegir sitio de acceso para realizar un TAVR no tendrán los resultados esperados.

El estudio US PIVOTAL ${ }^{14}$, con mayor cantidad de pacientes, tuvo resultados diferentes a los anteriores ECR. La mortalidad de cualquier causa al año de seguimiento se redujo significativamente en el grupo TAVR en comparación con RVAO. Estos resultados fueron consistentes al comparar los 9 subgrupos evaluados en el ECR, sobre todo en mujeres. A diferencia del estudio PARTNER, en el grupo TAVR no hubo mayor incidencia de stroke, pudiendo deberse este resultado a la naturaleza autoexpandible del dispositivo estudiado $^{29}$. Además, la incidencia de regurgitación paravalvular al año de seguimiento fue de $6,1 \%$, menor que la esperada y pudiendo atribuirse a la evaluación previa al procedimiento del anillo valvular realizada por tomografía computada y no por eco 3-D, relacionado sistemáticamente en distintas series con peores resultados ${ }^{20-22}$. Los autores realizaron un análisis exploratorio de los eventos cardiovasculares y cerebrovasculares mayores, encontrando diferencias significativas al año a favor de TAVR $(p=0,03)$, pero estos datos tienen que ser tomados como generadores de hipótesis ya que el tamaño del estudio no tenía poder muestral para sacar estas conclusiones y no fue diseñado con este objetivo. Si bien los autores sugieren en las conclusiones que la naturaleza menos invasiva del procedimiento y la posibilidad para el paciente de movilizarse más tempranamente hayan influenciado en los buenos resultados del estudio, el score STS PROMPT fue del 7,3\% y la mortalidad real del grupo RVAO a 30 días fue menor (4,5\%), pudiendo indicar la sobreestimación del score de riesgo como limitación al momento de analizar los resultados.

El estudio PARTNER 2 cohorte A demuestra que la TAVR de nueva generación, en centros de experiencia, es no inferior a la cirugía en pacientes con riesgo intermedio en términos de mortalidad o stroke a dos años de evolución ${ }^{15}$. Por otro lado se encontraron beneficios en el grupo TAVR al evaluar los puntos finales secundarios del estudio, incluyendo menor riesgo de sangrado, de injuria renal aguda, nueva fibrilación auricular, además de recuperación y alta más rápida (Tabla 5). Con respecto a los resultados ecocardiográficos, la frecuencia y severidad del reflujo aórtico paravalvular fue mayor en el grupo TAVR, algo que se relacionó directamente con aumento de la mortalidad. Esta limitación puede adjudicarse a que para elegir el tamaño protésico, la evaluación del anillo valvular se realizó por ECO 2D; posteriormente se han publicado numerosos reportes que demostraron que este método subvaloraba las dimensiones y que la tomografía axial multicorte debía ser el método de elección para para la evaluación previa a la selección del tamaño de prótesis en los procedimientos de TAVR ${ }^{10,20-22}$.

En cuanto a la revascularización coronaria concomitante en los pacientes evaluados en el PARTNER 2, hubo mayor necesidad de cirugía de bypass en el grupo RVAO comparado con TAVR $(14,5$ vs. 3,9\%) sin tener repercusión en cuanto a la incidencia de mortalidad o stroke. Estos resultados se contradicen con estudios clínicos previos que asociaron la revascularización concomitante en el grupo RVAO con un aumento en la mortalidad ${ }^{23,24}$.

$\mathrm{Al}$ analizar los dos estudios más grandes, el PARTNER 2 A y el SURTAVI, se evidencia rápidamente que la mortalidad a 24 meses, si bien tuvo la misma tendencia, fue menor en el segundo (11,4 en grupo TAVR-SURTAVI $v$ s. $11,6 \%$ en
RVAO-SURTAVI y 16,7 en TAVR-PARTNER vs. $18,0 \%$ en SAVR-PARTNER). Esto pudo deberse a que el sistema de calificación (score) de riesgo quirúrgico de los pacientes del SURTAVI fue menor, y siendo reconocido que estos scores tienen falencias importantes al no valorar rasgos como la fragilidad, discapacidad o enfermedades concomitantes, puede ser una sugerencia de que deben modificarse los mismos, por lo menos para valorar a estos grupos de pacientes de riesgos menores.

Una limitante importante en los estudios analizados fue la alta incidencia de abandonos y drop-outs en el grupo RVAO. Aunque no se presentaron diferencias importantes en cuanto al análisis de intención de tratar y efectivamente tratados finalmente ${ }^{15,16}$, estos sucesos hacen notar que aquellos pacientes a los que se decidió realizar una RVAO debieron ser cuidadosamente evaluados por el equipo quirúrgico para lograr una contención adecuada.

Por último, se realizó un análisis estadístico de los eventos adversos desde el procedimiento hasta los 30 días, como puede apreciarse en la Tabla 5, y de la mortalidad en el seguimiento, como se ve en la Tabla 6. En la primera de las tablas puede apreciarse como los 5 estudios tienen puntos en común claro, dándoles credibilidad a los mismos en la coincidencia. En todos salvo uno (STACCATO) la incidencia de injuria renal aguda fue significativamente mayor en el grupo RVAO, al igual que el sangrado severo y la fibrilación auricular. En cambio, el implante de marcapaso definitivo y las complicaciones vasculares severas fueron estadísticamente mayores en el grupo TAVR. Este análisis es importante para valorar y entender cuáles son las complicaciones esperables en el primer momento post quirúrgico en ambos tipos de procedimiento.

Con respecto al análisis de mortalidad, en la Tabla 6 se evalúan los 5 estudios a 30 días, 1 año y dos años de seguimiento de acuerdo a la información brindada en los ECR seleccionados. No se aprecian diferencias entre ambos grupos en ninguno de los tiempos de seguimiento, y la tendencia se mantiene a lo largo del tiempo. El resultado global a 2 años de los 3 ECR que brindan sus datos se relaciona directamente con lo previamente reportado, sin importar el peso específico que cada uno de los estudios tenga de forma individual.

\section{CONCLUSIONES}

La cirugía de reemplazo valvular aórtico sigue siendo de elección en pacientes con estenosis aórtica severa sintomática de riesgo bajo e intermedio en la República Argentina, y si bien los datos que se obtuvieron de este análisis no permiten establecer conclusiones definitivas, los resultados aquí observados sugieren que la indicación de TAVR en individuos con riesgo intermedio es posible, y que aún se necesita un mayor número de evidencias para una indicación precisa. La mayoría de los ECR demostraron que la TAVR es no inferior al RVAO en pacientes con estenosis aórtica sintomática y riesgo quirúrgico intermedio. La tendencia de estos estudios además muestra que, en determinadas poblaciones especiales, la TAVR realizada por acceso femoral es superior a la cirugía convencional, como en las mujeres, los pacientes frágiles y los ancianos ${ }^{41,42}$. Para ello es fundamental que el equipo médico especializado o heart team considere todas los aspectos posibles al momento de elegir entre RVAO o TAVR en pacientes con riesgo quirúrgico aumentado. Las guías de 
enfermedad valvular de la Sociedad Europea de Cardiología del año 2017 consideran aspectos clínicos, anatómicos, técnicos y condiciones cardíacas además de la enfermedad valvular aórtica para elegir el procedimiento apropiado para cada paciente de forma individual ${ }^{10}$.

Existen varias cuestiones pendientes para evaluar en el futuro: Primero, no existen análisis de costo efectividad en pacientes con riesgo intermedio. Si bien el TAVR tiene mayor costo que RVAO, ambos procedimientos requieren equipos entrenados y con experiencia, y la diferencia entre el costo de la prótesis podría saldarse con las diferencias de tiempos de internación y requerimientos de rehabilitación post quirúrgica. Un análisis de costos y beneficios de uno u otro procedimiento en nuestro país debe ser cuidadosamente medido. El valor individual del TAVR en Argentina parece excesivo; sin embargo los costos económicos de tiempo de hospitalización, posibles complicaciones y posterior recuperación de los pacientes sometidos a RVAO, sobre todo en pacientes frágiles, debe ser medido.

Segundo, la durabilidad de las prótesis valvulares todavía está en debate. Los estudios PARTNER previos ya comenzaron a publicar datos en donde no se identifica deterioro prematuro de la válvula protésica a 5 años de seguimiento ecocardiográfico ${ }^{38,39}$. En julio de 2017 se presentó en el Heart una exhaustiva revisión bibliográfica donde se evalúan 13 estudios y un total de 8914 pacientes con un segui-

\section{BIBLIOGRAFÍA}

1. Osnabrugge RL, Mylotte D, Head SJ, Van Mieghem NM, Nkomo VT, LeReun CM et al. Aortic stenosis in the elderly: Disease prevalence and number of candidates for transcatheter aortic valve replacement: $A$ meta-analysis and modeling study. J Am Coll Cardiol 2013;62:10021012.

2. Holmes DR Jr, MackMJ, Kaul S, Agnihotri A, Alexander KP, Bailey SR, et al. 2012 ACCF/AATS/SCAI/STS expert consensus document on transcatheter aortic valve replacement. J Am Coll Cardiol. 2012; 59:1200-54. 3. Consenso devalvulopatías SAC. VOL 83 SUPLEMENTO 2 JUNIO 2015 SAC.

4. Holmes DR Jr, Mack MJ, Kaul S, Agnihotri A, Alexander KP, Bailey SR, et al. 2012 ACCF/AATS/SCAI/STS expert consensus document on transcatheter aortic valve replacement. J Am Coll Cardiol. 2012; 59:1200-54.

5. Vahanian A, Alfieri O, Andreotti F, Antunes MJ, Baron-Esquivias G, Baumgartner $\mathrm{H}$, et al; Joint Task Force on the Management of Valvular Heart Disease of the European Society of Cardiology (ESC). Guidelines on the management of valvular heart disease (version 2012). Eur Heart J. 2012:33:2451-96.

6. Smith CR, Leon MB, Mack MJ, Miller DC, Moses JW, Svensson LG, et al; PARTNER Trial Investigators. Transcatheter versus surgical aortic-valve replacement in high-risk patients. N Engl J Med. 2011; 364:2187-98.

7. Kodali SK, Williams MR, Smith CR, Svensson LG, Webb JG, Makkar RR, et al; PARTNER Trial Investigators. Two-year outcomes after transcatheter or surgical aortic-valve replacement. N Engl J Med. 2012;366:1686-95.

8. Nkomo VT, Gardin JM, Skelton TN, Gottdiener JS, Scott CG, Enriquez-Sarano M. Burden of valvular heart diseases: a population based study. Lancet 2006;368:1005-11.

9. Nishimura RA, Otto CM, Bonow RO, Carabello BA, Erwin JP, Fleisher LA et al. 2017 AHA/ACC Focused Update of the 2014 AHA/ACC Guideline for the Management of Patients With Valvular Heart Disease: A Report of the American College of Cardiology/American Heart Association Task Force on Clinical Practice Guidelines. Circulation. 2017;135:e1159-e1195

10. Baumgartner H, FalkV, BaxJJ, De Bonis M, Hamm C, Holm PJ et al. 2017 ESC/EACTS Guidelines for the management of valvular heart disease: The Task Force for the Management of Valvular Heart Disease of the European Society of Cardiology (ESC) and the European Association for Cardio-Thoracic Surgery (EACTS). Eur Heart J. 2017 Aug 26, ehx391. miento medio entre 1,6 a 5 años que no evidencia deterioro estructural valvular, calculando una incidencia global de 28 eventos cada 10.000 pacientes/año (IC95\%: 2,46-73.44 cada 100 pacientes/año $)^{40}$. Además los autores encontraron que, entre aquellos que desarrollaron valvulopatía estructural, solo el $12 \%$ fue sometido a una reintervención (40\%). Sin embargo, de esto no se desprende que a mayor seguimiento las válvulas no tengan un deterioro más severo y estudios más prolongados en el tiempo son necesarios para saldar esta incógnita.

Tercero, la regurgitación aórtica paravalvular post implante percutáneo se relaciona con mal pronóstico solo cuando es moderada a severa ${ }^{20-22} \mathrm{y}$, aunque fue significativamente mayor en el grupo TAVR, en esta serie de pacientes la misma ocurrió en menos del $4 \%$ de los casos. Con el advenimiento de las nuevas tecnologías para la evaluación de las dimensiones del anillo valvular aórtico y los dispositivos autoexpandibles, esto podría modificarse en un futuro.

Finalmente, la incidencia de implante de marcapaso definitivo sigue siendo alta en algunas series, y a pesar que esto no se ha relacionado con un incremento en la mortalidad, es una limitación importante al momento de querer ampliar las indicaciones de TAVR a pacientes con riesgo intermedio o bajo. De cualquier modo, todos los ECR evaluados fueron en pacientes de edades avanzadas, por lo cual no existen datos en poblaciones de menor edad.

11. Rey-Ares L, Alcaraz A, Pichón-Riviere A, Augustovski F, García MartíS, Bardach Ay col. Reemplazo valvular aórtico percutáneo para el tratamiento deestenosis severa. Documentos de Evaluación de Tecnologías Sanitarias, Informe de Respuesta Rápida N³41, Buenos Aires, Argentina. Mayo 2014. Disponible en www.iecs.org.ar

12. Nielsen HH, Klaaborg KE, Nissen H, Terp K, Mortensen PE, Kjeldsen BJ et al. A prospective, randomized trial of transapical transcatheter aortic valve implantation vs. surgical aortic valve replacement in operable elderly patients with aortic stenosis: the STACCATO trial. Eurolntervention 2012;8:383-9.

13. Thyregod HGH, Steinbrüchel DA, Ihlemann N, Nissen H, Kjeldsen BJ, Petursson P et al. Transcatheter Versus Surgical Aortic Valve Replacement in Patients With Severe Aortic Valve Stenosis 1-Year Results From the All-Comers NOTION Randomized Clinical Trial. J Am Coll Cardiol 2015;65:2184-94

14. Adams DH, Popma JJ, Reardon MJ, Yakubov SJ, Coselli JS, Deeb GM et al. U.S. CoreValve Clinical Investigators. Transcatheter aortic-valve replacement with a self expanding prosthesis. N Engl J Med 2014,370:1790-8

15. Leon MB, Smith CR, MackMJ, MakkarRR, Svensson LG, KodaliSKetal.PARTNER2 Investigators. Transcatheter or Surgical Aortic-Valve Replacement in Intermediate-Risk Patients. N Engl J Med 2016;374:1609-20.

16. Reardon MJ, Van Mieghem NM, Popma JJ, Kleiman NS, Søndergaard L, Mumtaz M et al. for the SURTAVI Investigators. Surgical or Transcatheter Aortic-Valve Replacement in Intermediate-Risk Patients. N Engl J Med. 2016 Apr 28:374(17):1609-20

17. AP Kappetein, SJ Head, P Genereux Piazza N, van Mieghem NM, Blackstone EH et al. Updated standardized endpoint definitions for transcatheter aortic valve implantation: the Valve Academic Research Consortium-2 consensus document. J Am Coll Cardiol 2012;60:1438-54

18. Green P, Woglom AE, Genereux P, Daneault B, Paradis JM, Schnell S et al. The impact of frailty status on survival after transcatheter aortic valve replacement in older adults with severe aortic stenosis: a single-center experience. JACC Cardiovasc Interv 2012:5:974-81.

19. O'Brien SM, Shahian DM, Filardo G, Filardo G, Ferraris VA, Haan CK et al. The Society of Thoracic Surgeons 2008 cardiac surgery risk models. 2. Isolated valve surgery. Ann Thorac Surg 2009; 88: Suppl 1: S23-42. 
20. Lehmkuhl L, Foldyna B, Von Aspern K, Lücke C, Grothoff M, Nitzsche S et al. Inter-individual variance and cardiac cycle dependency of aortic root dimensions and shape as assessed by ECG-gated multi-slice computed tomography in patients with severe aortic stenosis prior to transcatheter aortic valve implantation: is it crucial for correct sizing? Int I Cardiovasc Imaging 2013;29:693-703.

21. Binder RK, Webb JG, Willson AB, Urena M, Hansson NC, Norgaard BL et al. The impact of integration of a multidetector computed tomography annulus area sizing algorithm on outcomes of transcatheter aortic valve replacement: a prospective, multicenter, controlled trial. J Am Coll Cardiol 2013;62:431-8

22. Mylotte D, Dorfmeister M, Elhmidi Y, Mazzitelli D, Bleiziffer S, Wagner A et al. Erroneous measurement of the aortic annular diameterusing 2-dimensional echocardiography resulting in inappropriate CoreValve size selection: a retrospective comparison with multislice computed tomography. J Am Coll Cardiol Intv 2014;7: 652-61.

23. Brennan $M M, E d w a r d s F H, Z$ Zhao Y, O'Brien SM, Douglas PS, PetersonED. Longterm survival after aortic valve replacementamong high-riskelderly patients in the United States: insights from the Society of Thoracic Surgeons AdultCardiac Surgery Database, 1991 to 2007. Circulation 2012; 126: 1621-9.

24. Holzhey D, Mohr FW, Walther T, Möllmann H, Beckmann A, Kötting J et al. Current results of surgical aortic valve replacement: Insights from the German Aortic Valve Registry. Ann Thorac Surg 2016; 101: 658-66.

25. Thourani VH, Suri RM, Gunter RL, Sheng S, O'Brien SM, Ailawadi G et al. Contemporary real-world outcomes of surgical aortic valve replacement in 141,905 low-risk, intermediate-risk, and high-risk patients. Ann Thorac Surg 2015; 99: 55-61.

26. Mack MJ, Brennan JM, Brindis R, Carroll J, Edwards F, Grover Fet al. Outcomes following transcatheter aortic valve replacement in the United States. JAMA 2013; 310: 2069-77.

27. Popma JJ, Adams DH, Reardon MJ, Yakubov SJ, Kleiman NS, Heimansohn D et al. Transcatheter aortic valve replacement using a self-expanding bioprosthesis in patients with severe aortic stenosis at extreme risk for surgery. J Am Coll Cardiol 2014; 63: 1972-81.

28. Leon MB, Smith CR, MackM, Miller DC, Moses JW, Svensson LG et al. Transcatheter aortic-valve implantation for aortic stenosis in patients who cannot undergo surgery. N Engl J Med 2010; 363: 1597-607.

29. Smith CR, Leon MB, MackMJ, MillerDC, Moses JW, Svensson LG etal. Transcatheter versus surgical aortic-valve replacement in high-risk patients. N Engl J Med 2011; 364:2187-98.

30. Siontis GC, Praz F, Pilgrim T, Mavridis D, Verma S, Salanti G et al. Transcatheter aortic valve implantation vs. surgical aortic valve replacement for treatment of severe aortic stenosis: a meta- analysis of randomized trials. Eur Heart J 2016; 37: 3503-12.

31. Gargiulo G, Sannino A, Capodanno D, Barbanti M, Buccheri S, Perrino C et al. Transcatheter Aortic Valve Implantation Versus Surgical Aortic Val- ve Replacement A Systematic Review and Meta-analysis. Ann Intern Med. 2016:165:334-344

32. Siemieniuk RA, Agoritsas T, Manja V, Devii T, Chang Y, Bala MM et al. Transcatheter versus surgical aortic valve replacement in patients with severe aortic stenosis at low and intermediate risk: systematic review and meta-analysis. BMJ 2016;354:i5130.

33. Khan AR, Khan S, Riaz H, Luni FK, Simo H, Bin Abdulhak A et al. Efficacy and safety of transcatheter aortic valve replacement in intermediate surgical risk patients: A systematic review and meta-analysis. Catheter Cardiovasc Interv. 2016 Nov 15;88(6):934-944.

34. Panchal HB, Ladia V, DesaiS, Shah T, Ramu V. A meta-analysis of mortality and major adverse cardiovascular and cerebrovascular events following transcatheter aortic valve implantation versus surgical aortic valve replacementfor severe aortic stenosis. Am J Cardiol. 2013 Sep 15;112(6):850-60.

35. Kondur A, Briasoulis A, Palla M, Penumetcha A, Mallikethi-Reddy S, Badhe$k a$ A et al. Meta-Analysis of Transcatheter Aortic Valve Replacement Versus Surgical Aortic Valve Replacement in Patients With Severe Aortic Valve Stenosis. Am J Cardiol. 2016 Jan 15:117(2):252-7.

36. Vandvik PO, Otto CM, Siemieniuk RA, Bagur R, Guyatt GH, Lytvyn L et al. Transcatheter or surgical aortic valve replacement for patients with severe, symptomatic, aortic stenosis at low to intermediate surgical risk: a clinical practice guideline. BMJ. 2016 Sep 28;354:i5085.

37. MoatNE, Ludman P, de Belder MA, Bridgewater B, Cunningham AD, Young $C P$ et al. Long-term outcomes after transcatheter aortic valve implantation in high risk patients with severe aortic stenosis: the U.K. TAVI (United Kingdom Transcatheter Aortic Valve Implantation) Registry. J Am Coll Cardiol 2011; 58: 2130-8.

38. Kapadia SR, Leon MB, Makkar RR, Tuzcu EM, Svensson LG, Kodali S et al. 5-Year outcomes of transcatheter aortic valvereplacement compared with standard treatment for patients with inoperable aortic stenosis (PARTNER 1): a randomised controlled trial. Lancet 2015; 385: 2485-91.

39. Mack MJ, Leon MB, Smith CR, Miller DC, Moses JW, Tuzcu EM et al. 5-Year outcomes of transcatheter aortic valve replacement or surgical aortic valvereplacementfor high surgical risk patients with aorticstenosis (PARTNER 1): a randomised controlled trial. Lancet 2015;385: 2477-84.

40. Foroutan F, Guyatt GH, Otto CM, Siemieniuk RA, Schandelmaier S, Agoritsas $T$ et al. Structural valve deterioration after transcatheter aortic valve implantation. Heart. 2017 Jul 6. pii: heartjnl-2017-311329 [Epub ahead of print]

41. Green P, Woglom AE, Genereux P, Daneault B, Paradis JM, Schnell S et al. The impact of frailty status on survival after transcatheter aortic valve replace ment in older adults with severe aortic stenosis: a single-center experience. JACC Cardiovasc Interv 2012; 5: 974-81.

42. Hermiller JB JI, Yakubov SJ, Reardon MJ, Deeb GM4, Adams DH5, Afilalo $J$ et al. Predicting early and late mortality after transcatheter aortic valve replacement. J Am Coll Cardiol 2016; 68: 343-52. 